\title{
Reactive nodular fibrous pseudotumor involving the gastrointestinal tract and mesentery: A case report and review of the literature
}

\author{
FEI YAN ${ }^{1}$, YANLI MA ${ }^{1}$, JIANHAI SUN ${ }^{1}$ and PENGCHENG ZHU ${ }^{2}$ \\ ${ }^{1}$ Department of Oncology, Zhongshan Hospital of Hubei Province, Wuhan, Hubei 430000; ${ }^{2}$ Institute of Pathology, \\ Tongji Hospital, Tongji Medical College, Huazhong University of Science and Technology, Wuhan, Hubei 430030, P.R. China
}

Received April 1, 2014; Accepted December 12, 2014

DOI: $10.3892 / 01.2015 .2882$

\begin{abstract}
Reactive nodular fibrous pseudotumor (RNFP) is a tumor-like lesion that is characterized by reactive fibroblast/myofibroblast proliferation within collagenic hyalinized stroma, due to its association with injury or inflammation. The current study describes the case of a 60-year-old female with a history of abdominal surgery and abdominal pain. Upon laparoscopy, multiple nodules attached to the outer layer of the colon and mesentery were identified, and therefore, complete surgical excision was performed. Macroscopically, the nodules were well-circumscribed, firm, tan-white in color and ranged in size between $2.0-10.0 \mathrm{~cm}$ at the greatest dimension. Microscopically, the nodules were composed of spindle and stellate cells in a dense collagenic hyalinized background with sparse lymphocytic infiltration. Immunohistochemical analysis demonstrated positive staining for vimentin, smooth muscle actin and cluster of differentiation (CD) 117, and focally-positive keratin staining with AE1/AE3; however, no staining was observed for gastrointestinal stromal tumor 1, CD34, S-100, anaplastic lymphoma kinase or $\beta$-catenin. Therefore, it was proposed that the lesion may be most accurately described as an RNFP. The current study reports a rare case of RNFP, emphasizing its histopathological features and differential diagnoses to promote an improved and broader understanding of this poorly understood condition.
\end{abstract}

\section{Introduction}

Reactive nodular fibrous pseudotumor (RNFP) is described as a rare benign tumor-like lesion (1-3), which was first reported by Yantiss et al in 2003 (1). According to the limited literature,

Correspondence to: Dr Pengcheng Zhu, Institute of Pathology, Tongji Hospital, Tongji Medical College, Huazhong University of Science and Technology, 1095 Jiefang Dadao, Wuhan, Hubei 430030, P.R. China

E-mail: zhupengcheng@mails.tjmu.edu.cn

Key words: reactive nodular fibrous pseudotumor, gastrointestinal tract, mesentery, differential diagnosis
RNFP predominantly occurs in the gastrointestinal (GI) tract and peritoneal regions with a male predominance (male/female ratio, 14/5) in adults. Macroscopically, it appears as a single mass or multiple nodules attached to the outer layer of the bowel wall (3), mesentery or omentum (2). Microscopically, the lesion is composed of spindle or stellate cells resembling fibroblasts/myofibroblasts enmeshed in a collagenous matrix, which is typically hyalinized or keloidal in nature $(1,4)$. The fact that the lesion often presents with multiple intra-abdominal masses evidently causes clinical concern for malignancy, and complete resection remains a routine treatment. RNFP shows a good prognosis without signs of recurrence or metastasis, no cases of RNFP recurrence or metastasis have been reported in the literature. The present study reports and analyzes a case of RNFP involving the mesentery and greater omentum in a 60-year-old female patient, with the aim of improving the characterization of RNFP, and identifying distinguishing features of the lesion to improve its differential diagnosis from other neoplasms and non-neoplastic lesions involving this anatomical region. The present study was approved by the ethics committee of Zhongshan Hospital of Hubei Province (Wuhan, China) and written informed consent was obtained from the patient.

\section{Case report}

In September 2012, a 60-year-old female patient presented to the Department of Oncology, Zhongshan Hospital of Hubei Province (Wuhan, China) with abdominal pain that had gradually developed over six months following abdominal surgery for leiomyoma of the uterus. Upon laparoscopy, multiple nodules were identified throughout the mesentery, greater omentum and serosal surface of the colon, ranging in size between $2.0-10.0 \mathrm{~cm}$ at the largest dimension. Due to the presence of multiple nodules diffused throughout the abdominal cavity, the lesion was diagnosed as a metastatic malignant tumor. Thus, a partial colectomy was performed and all masses were resected prior to macroscopic, histological and immunohistochemical examination of the specimens.

The resected specimens were fixed in $10 \%$ formalin, embedded in paraffin, sectioned and stained with hematoxylin and eosin in accordance with routine procedures, and 
immunostaining was performed on $4-\mu$ m-thick sections using the standard avidin-biotin complex technique. A panel of antibodies (Table I) was used to evaluate the tumor samples for the presence of smooth muscle [Desmin and smooth muscle actin (SMA)], fibroblastic/myofibroblastic (SMA), schwannian (S-100) and epithelial cell (AE1/AE3) differentiation, and various immunohistochemical markers typically expressed in GI stromal tumors [GIST; discovered on GIST (DOG) 1, cluster of differentiation (CD) 117, CD34], inflammatory myofibroblastic tumors [anaplastic lymphoma kinase (ALK)], aggressive fibromatosis ( $\beta$-catenin) and immunoglobulin (Ig) G4-associated disease (IgG4, IgG).

Sections of healthy colon tissue (Maixin Bio, Fuzhou, China) served as the positive controls for the expression of vimentin, SMA, desmin, AE1/AE3, S-100, DOG1, CD34 and CD117, and an ALK-positive anaplastic large cell lymphoma cell line (Maixin Bio) served as the positive control for ALK staining. Negative controls were performed by replacing the primary antibodies with saline.

Macroscopically, the specimens were well-circumscribed, rubbery to hard, white-tan-colored nodules ranging between $2.0-10.0 \mathrm{~cm}$ at the largest dimension, while the cut surface of the lesions was solid and homogeneous (Fig. 1A). The nodules predominantly involved the outer layer of the colon, mesentery and omentum, but were partially associated with the transmural extension of the colon. Microscopically, the nodules were composed of sparse wavy spindle or stellate cells within hyalinized keloid-like collagen (Fig. 1B and C), although certain nodules contained a more cellular peripheral zone composed of active fibroblasts (Fig. 1D) or were surrounded by an inflammatory infiltrate of mononuclear lymphoid cells.

Immunohistochemically, the spindle or stellate cells were positive for vimentin, SMA (Fig. 1E) and CD117, focally positive for AE1/AE3 (Fig. 1F) and desmin expression, and demonstrated no DOG1, CD34, S-100 or $\beta$-catenin expression. In addition, high counts of IgG4-positive plasma cells were not detected and the ratio of $\mathrm{IgG} 4 / \mathrm{IgG}$-positive cells was determined as $<40 \%$.

The patient was regularly followed up, and at 12 months post-resection the patient remains alive and in good health with no evidence of recurrence or metastatic disease. The follow-up continues at approximately eight-week intervals at present.

\section{Discussion}

RNFP was initially described in a series by Yantiss et al (1), and is characterized by fibroblastic/myofibroblastic spindle or stellate cells with a hyalinized collagenous background and a lack of atypia and mitosis. RNFP is considered to be a reactive benign lesion that is associated with previous surgical procedures or inflammatory disorders (4). Immunohistochemically, the spindle cells are typically positive for vimentin, SMA, desmin and AE1/AE3 expression, and demonstrated no DOG1, CD34 or S-100 expression. Previous observations of CD117 and AE1/AE3 expression in RNFP $(1,4)$ appear to conflict with the results of the present study, which identified focal positive CD117 and peripheral cellular zonal AE1/AE3 staining. Yantiss et al (1) observed CD117 expression in four out of five cases of RNFP, while Daum et al (4) confirmed no CD117
Table I. Antibodies and dilutions used in the evaluation of reactive nodular fibrous pseudotumor.

\begin{tabular}{llc}
\hline Antibody & Dilution & Supplier \\
\hline Vimentin & $1: 20$ & Dako \\
AE1/AE3 & $1: 20$ & Dako \\
CD117 & $1: 40$ & Dako \\
SMA & $1: 100$ & Dako \\
DOG1 & $1: 50$ & Leica Biosystems \\
CD34 & $1: 20$ & Dako \\
Desmin & $1: 140$ & Dako \\
S-100 & $1: 1000$ & Dako \\
ALK & $1: 10$ & Dako \\
$\beta$-catenin & $1: 400$ & Dako \\
IgG4 & $1: 500$ & Leica Biosystems \\
IgG & $1: 250$ & Zymed \\
\hline
\end{tabular}

For all antibodies, heat-induced antigen retrieval was used to improve staining prior to immunohistochemistry. $\mathrm{CD}$, cluster of differentiation; SMA, smooth muscle actin; DOG1, discovered on GIST 1; ALK, anaplastic lymphoma kinase; IgG, immunoglobulin G.

expression among eight cases of RNFP. However, analysis of the relevant literature indicates that these conflicting observations may be the result of methodological differences (Table II), such as different antibody clones or antigen retrieval details. Furthermore, the immunohistochemical profile of the present patient was similar to the characteristic fibroblastic/myofibroblastic differentiation of RNFP (5). Considering the specific marker expression profiles of RNFP spindle cells, including the coexpression of vimentin and low molecular-weight cytokeratin, determined by AE1/AE3 expression, it has been proposed that the cells may be derived from multipotential subserosal cells (4).

Cases of RNFP arising within the abdominal cavity $(6,7)$, including the GI tract (3), mesentery and retroperitoneum, are rare but important due to their propensity to be misdiagnosed as more aggressive types of tumor, such as metastatic malignant neoplasm, primary GIST or inflammatory myofibroblastic (IMF) tumor. The case presented in the present study was initially suspected to be a metastatic malignant tumor due to the presence of multiple diffuse nodules within the abdominal cavity. However, histopathological analysis of the lesion determined rare mitotic figures and no nuclear atypia or necrosis. Based upon these clinical and histopathological features, it is proposed that the lesion was a cohesive case of distinct tumor-like fibroblastic/myofibroblastic proliferation that represents an acute post-operative response to surgery.

A recent case series described four patients with RNFP of the GI tract that possessed abundant IgG4-positive plasma cells, indicating that RNFP may form part of the IgG4-associated disease spectrum in specific cases $(8,9)$. However, in the present case, high counts of IgG4-positive plasma cells were not observed and the ratio of $\mathrm{IgG} 4 / \mathrm{IgG}$ was $<40 \%$.

Certain spindle cell lesions arising from the GI tract should be distinguished from RNFP upon diagnosis. GIST, a common mesenchymal tumor in this anatomical region (10), is typically 
Table II. Summary of the reported cases of reactive nodular fibrous pseudotumor.

\begin{tabular}{|c|c|c|c|}
\hline First author (reference) & Year & Cases, $\mathrm{n}$ & Positive expression, protein (n) \\
\hline Yantiss et al (1) & 2003 & 5 & $\begin{array}{l}\text { Vim (5); CD117 (4); SMA (3); } \\
\text { CD34 (0); CK (0); ALK (ND) }\end{array}$ \\
\hline Daum et al (4) & 2004 & 8 & $\begin{array}{l}\text { Vim (7); CD117 (0); SMA (8); } \\
\text { CD34 (0); CK (6); ALK (ND) }\end{array}$ \\
\hline Saglam et al (2) & 2005 & 1 & $\begin{array}{l}\operatorname{Vim}(1) ; \text { CD117 (ND); SMA (1); } \\
\text { CD34 (1); CK (1); ALK (ND) }\end{array}$ \\
\hline Gauchotte et al (7) & 2009 & 1 & $\begin{array}{l}\operatorname{Vim}(1) ; \operatorname{CD} 117(0) ; \operatorname{SMA}(1) \\
\text { CD34 (0); CK (1); ALK (0) }\end{array}$ \\
\hline McAteer et al (6) & 2012 & 1 & $\begin{array}{l}\text { Vim (1); CD117 (0); SMA (1); } \\
\text { CD34 (0); CK (1); ALK (0) }\end{array}$ \\
\hline
\end{tabular}

Vim, vimentin; CD, cluster of differentiation; SMA, smooth muscle actin; CK, cytokeratin; ALK, anaplastic lymphoma kinase; ND, not done.
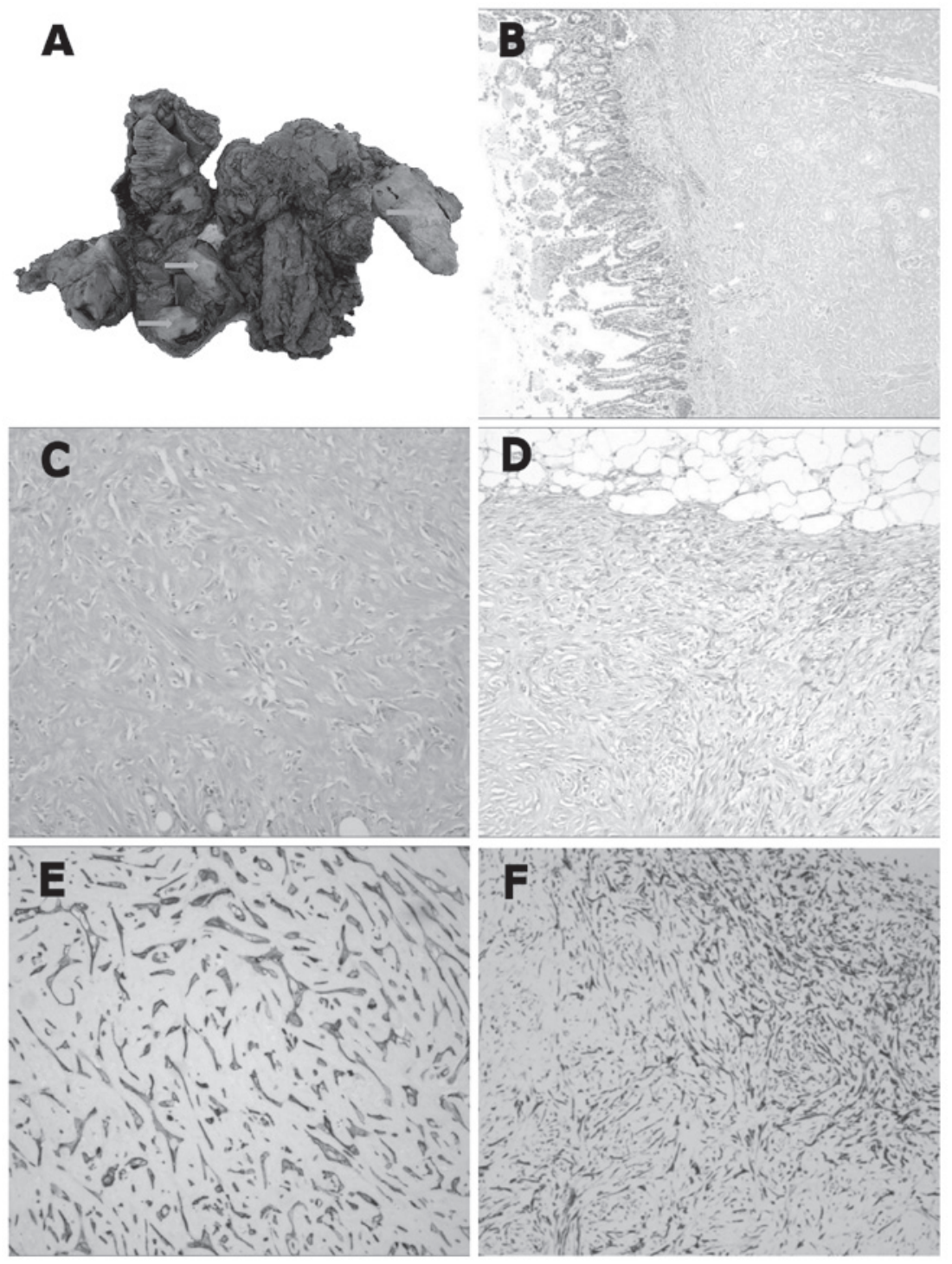

Figure 1. (A) Macroscopic image of the surgical specimen demonstrating multiple tan-white nodules (arrows) of varying sizes attached to outer layer wall of the colon and mesentery. (B) Reactive nodular fibrous pseudotumor (RNFP) of the colon infiltrating and replacing the muscularis propria and encroaching on the submucosa (magnification, x100). (C) RNFP were composed of proliferative spindle and stellate cells resembling fibroblasts/myofibroblasts in a dense collagenic hyalinized background (magnification, $x 400$ ), with (D) specific RNFP nodules containing a more cellular peripheral zone composed of active fibroblasts. (magnification, x200). (E) Diffuse strong immunohistochemical staining for smooth muscle actin (magnification, x400) and (F) focal AE1/AE3 expression, particularly in the peripheral cellular zone (magnification, x200; hemaoxylin and eosin staining). 
more cellular than RNFP and is immunopositive for DOG1, CD117 and CD34 (11,12). Although immunopositivity of CD117 and CD34 are the most valuable factors in the diagnosis of GIST, previous studies have demonstrated that DOG1, compared with CD117, may be a more specific and sensitive marker for GIST (13), particularly when RNFP and GIST are each positive for CD117 expression. In addition, calcifying fibrous pseudotumor $(14,15)$, which is generally more cellular than RNFP, including a mixture of granulocytes, plasma cells and infiltrating lymphocytes, frequently presents with psammomatous or dystrophic calcifications and is typically positive for CD34, but exhibits no SMA or desmin expression. Furthermore, IMF $(16,17)$ is another rare tumor that may be located in the mesentery or the retroperitoneum. IMF is a hypercellular tumor composed of loosely arranged fascicles of plump spindle cells with abundant, densely eosinophilic cytoplasm enmeshed within a variably collagenous, edematous or myxoid stroma. Additionally, IMF contains an active inflammatory infiltrate composed of plasma cells and lymphocytes that are closely associated with the tumor cells, in contrast to the mononuclear cell infiltrate of RNFP, which is typically sparse and patchy. Immunohistochemically, ALK-positive staining may also facilitate in the differential diagnosis of RNFP (18). Therefore, metastatic tumors in GI tract may be distinguished from RNFP by patient history and histopathological features.

Non-neoplastic spindle cell lesions that should also be distinguished from RNFP include aggressive fibromatosis (19), nodular fasciitis (20) and sclerosing mesenteritis (21). Thus, RNFP may be distinguished from mesenchymal lesions of the abdomen and GI tract by clinicopathological features and biological potential.

To conclude, RNFP is a post-operative or post-inflammatory lesion that is increasingly recognized in the differential diagnosis of primary and metastatic GI tumors. Therefore, it is important to differentiate RNFP from similar lesions with more aggressive phenotypes, as RNFP may be managed definitively with local resection and surgical follow-up.

\section{References}

1. Yantiss RK, Nielsen GP, Lauwers GY and Rosenberg AE: Reactive nodular fibrous pseudotumor of the gastrointestinal tract and mesentery: a clinicopathologic study of five cases. Am J Surg Pathol 27: 532-540, 2003.

2. Saglam EA, Usubütün A, Kart C, Ayhan A and Küçükali T: Reactive nodular fibrous pseudotumor involving the pelvic and abdominal cavity: a case report and review of literature. Virchows Arch 447: 879-882, 2005.

3. Yin SS, Zhang L, Cziffer-Paul A, Divino CM and Chin E: Reactive nodular fibrous pseudotumor presenting as a small bowel obstruction. Am Surg 77: 790-791, 2011.
4. Daum O, Vanecek T, Sima R, et al: Reactive nodular fibrous pseudotumors of the gastrointestinal tract: report of 8 cases. Int J Surg Pathol 12: 365-374, 2004.

5. Barak S, Wang Z and Miettinen M: Immunoreactivity for calretinin and keratins in desmoid fibromatosis and other myofibroblastic tumors: a diagnostic pitfall. Am J Surg Pathol 36: 1404-1409, 2012.

6. McAteer J, Huaco JC, Deutsch GH and Gow KW: Torsed reactive nodular fibrous pseudotumor in an adolescent: case report and review of the literature. J Pediatr Surg 47: 795-798, 2012.

7. Gauchotte G, Bressenot A, Serradori T, Boissel P, Plenat F and Montagne K: Reactive nodular fibrous pseudotumor: a first report of gastric localization and clinicopathologic review. Gastroenterol Clin Biol 33: 1076-1081, 2009.

8. Carruthers MN, Stone JH and Khosroshahi A: The latest on IgG4-RD: a rapidly emerging disease. Curr Opin Rheumatol 24: 60-69, 2012.

9. Chetty R, Serra S, Gauchotte G, Märkl B and Agaimy A: Sclerosing nodular lesions of the gastrointestinal tract containing large numbers of IgG4 plasma cells. Pathology 43: 31-35, 2011.

10. Liu FY, Qi JP, Xu FL and Wu AP: Clinicopathological and immunohistochemical analysis of gastrointestinal stromal tumor. World J Gastroenterol 12: 4161-4165, 2006.

11. Novelli M, Rossi S, Rodriguez-Justo M, et al: DOG1 and CD117 are the antibodies of choice in the diagnosis of gastrointestinal stromal tumours. Histopathology 57: 259-270, 2010.

12. Lee CH, Liang CW and Espinosa I: The utility of discovered on gastrointestinal stromal tumor 1 (DOG1) antibody in surgical pathology - the GIST of it. Adv Anat Pathol 17: 222-232, 2010.

13. Espinosa I, Lee CH, Kim MK, et al: A novel monoclonal antibody against DOG1 is a sensitive and specific marker for gastrointestinal stromal tumors. Am J Surg Pathol 32: 210-218, 2008.

14. Hill KA, Gonzalez-Crussi F and Chou PM: Calcifying fibrous pseudotumor versus inflammatory myofibroblastic tumor: a histological and immunohistochemical comparison. Mod Pathol 14: 784-790, 2001.

15. Fetsch JF, Montgomery EA and Meis JM: Calcifying fibrous pseudotumor. Am J Surg Pathol 17: 502-508, 1993.

16. Coffin CM,Watterson J, Priest JR and DehnerLP: Extrapulmonary inflammatory myofibroblastic tumor (inflammatory pseudotumor). A clinicopathologic and immunohistochemical study of 84 cases. Am J Surg Pathol 19: 859-872, 1995.

17. Shatzel J, Wooten K, Ankola A, Cheney RT, Morrison CD and Skitzki JJ: Inflammatory myofibroblastic tumor of the mesentery: a clinical dilemma. Int J Clin Oncol 17: 380-384, 2012.

18. Cook JR, Dehner LP, Collins MH, et al: Anaplastic lymphoma kinase (ALK) expression in the inflammatory myofibroblastic tumor: a comparative immunohistochemical study. Am J Surg Pathol 25: 1364-1371, 2001.

19. Ferenc T, Wronski JW, Kopczyński J, et al: Analysis of APC, alpha-, beta-catenins, and $\mathrm{N}$-cadherin protein expression in aggressive fibromatosis (desmoid tumor). Pathol Res Pract 205: 311-324, 2009.

20. Montgomery EA and Meis JM: Nodular fasciitis. Its morphologic spectrum and immunohistochemical profile. Am J Surg Pathol 15: 942-948, 1991.

21. Montgomery E, Torbenson MS, Kaushal M, Fisher C and Abraham SC: Beta-catenin immunohistochemistry separates mesenteric fibromatosis from gastrointestinal stromal tumor and sclerosing mesenteritis. Am J Surg Pathol 26: 1296-1301, 2002. 